\title{
Daridorexant: A new medication for insomnia in older adults?
}

Jennifer Rose V. Molano, MD

First published February 24, 2020, DOI: http://dx.doi.org/10.1212/WNL.0000000000009489

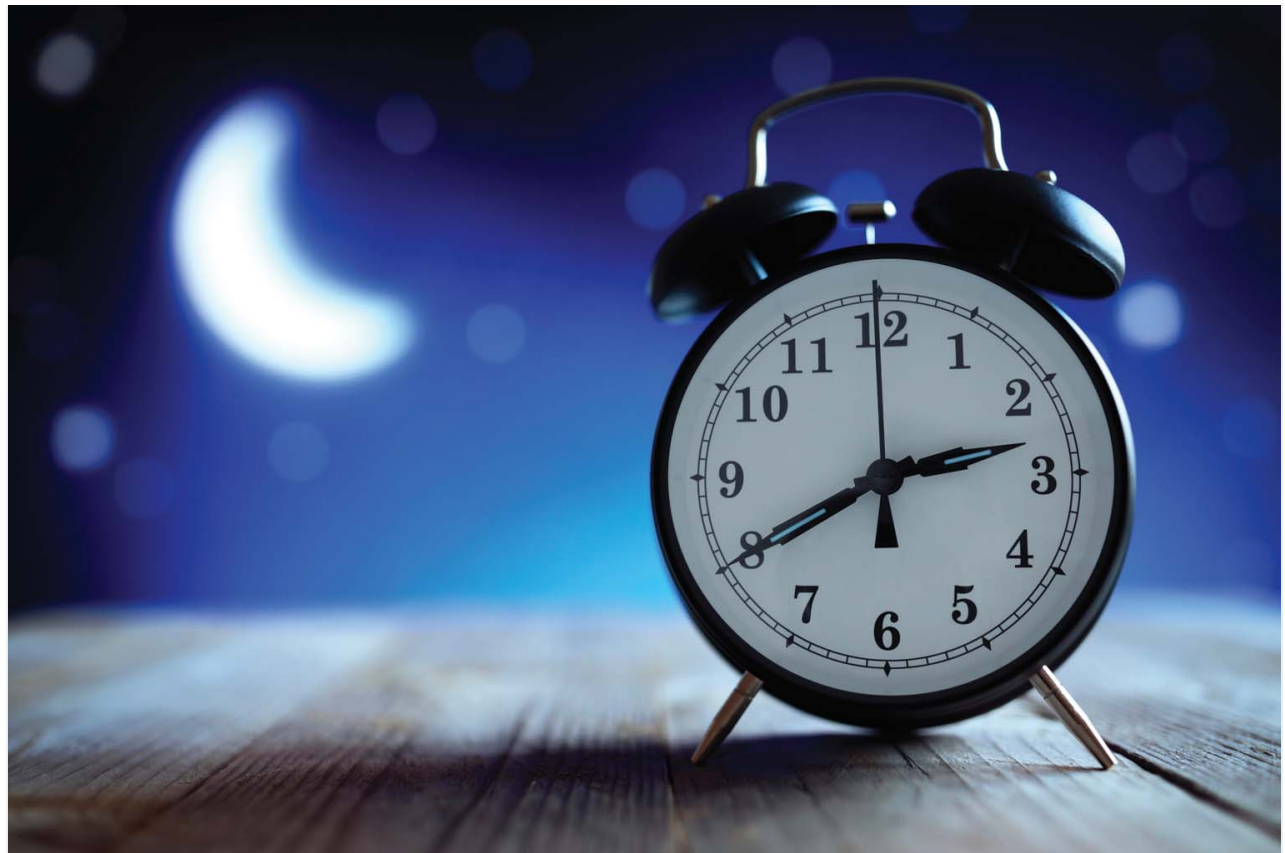

\section{What was the study and why is it important?}

In this issue of Neurology ${ }^{\circledR}$, the authors of this study determined whether daridorexant, a new sleep medication, was safe and effective in treating insomnia for adults $>65$ years of age. ${ }^{1}$ Insomnia is difficulty falling asleep or staying asleep that may interfere with daytime functioning, including an increased risk for falls, mood disorders such as depression, and decreased quality of life. ${ }^{2,3}$ It can be seen in about half of older adults. There are treatments, but commonly used medications for insomnia in older adults can increase the risk of thinking problems and falls. ${ }^{2,3}$

\section{How was the study done?}

In this study, participants reported that they usually took $>30$ minutes to fall asleep, woke up for $>30$ minutes after falling asleep, and had a total sleep time of $<61 / 2$ hours at least 3 times a week over 3 months. People could not join the study if they had known conditions that interfered with sleep. These included obstructive sleep apnea, restless legs syndrome, too much caffeine use, daytime naps $>1$ hour, and jet lag, among others.

The researchers studied 58 people who fit these rules. About two-thirds were women. First, they collected information about their usual sleep. The researchers used an electronic sleep diary and a 2-night sleep study. After this, participants were randomly assigned to a group. They received either a specific dose of the study medication $(5,10,25$, or $50 \mathrm{mg}$ daviorexant) or a placebo (sugar pill). They did not know which medication they were receiving. They took the pill 30 minutes before bedtime and at least 2 hours after dinner.

The researchers used sleep diaries and sleep study measurements to determine how well each treatment worked. They also did safety measurements to see whether the medication caused any problems in these older people.
Related Articles

Daridorexant, a new dual orexin receptor antagonist, in elderly subjects with insomnia disorder

http://dx.doi.org/10.1212/ WNL.0000000000009489 
The researchers mostly used the sleep studies to decide how well the medication worked. They measured how long it took people to fall asleep. They also looked at how much time people were awake after first falling asleep.

\section{What did the study show?}

Daridorexant allowed people to fall asleep faster and to stay asleep longer. Higher doses worked better than lower doses. When they compared results to the results of people who were given the placebo (sugar pill), they found that daridorexant doses $\geq 10 \mathrm{mg}$ clearly worked better than placebo. This is the best way to prove that a medication works. The differences were not small. It appeared that there were important improvements in both the ability to fall asleep more quickly and the ability to stay asleep. The participants also reported getting better sleep in their sleep diaries.
The medication was noted to have some possible side effects. These included fatigue, cold-like symptoms, walking difficulties, and headache. High doses especially caused more fatigue.

\section{What are the next steps?}

The results of this study showed that daridorexant may potentially be effective and safe in treating insomnia in older adults. This was a small study with only 58 participants. The next step will be to do a study including more people. This will give a better idea of how well daridorexant works and how safe it is. It is also important to compare daridorexant to other insomnia treatments. Future studies also should determine whether there are long-term effects of the medication such as thinking difficulties or risk for falls. 


\section{About daridorexant}

\section{How does daridorexant work to treat insomnia?}

Daridorexant blocks the action of a brain chemical called orexin. Orexin is a normal brain chemical that acts to help people stay awake. Therefore, blocking the action of orexin in the brain may help to promote sleep. ${ }^{2,3}$

\section{How does daridorexant work differently from other common medications for insomnia?}

Daridorexant acts on different brain systems than some other common insomnia medications. For example, many such as zolpidem (Ambien) work on the GABA brain chemical system. GABA is an important brain chemical that promotes sleep. ${ }^{2,3}$ Other medications work on different brain chemicals associated with sleep such as melatonin and histamine.

\section{How much sleep should an older adult get?}

The National Sleep Foundation recommends 7 to 8 hours of sleep for adults $>65$ years of age. ${ }^{4}$

\section{What happens to sleep as we get older?}

As we get older, we tend to spend more time in the lighter stages of sleep and less time in deeper sleep. We also have more nighttime awakenings. ${ }^{2,3,5}$ Much of this is a normal part of aging. However, underlying medical conditions, medications, and poor sleep habits often contribute to the symptoms of insomnia. ${ }^{2,5}$

\section{What is the general approach to the treatment of insomnia?}

Many factors may contribute to insomnia. ${ }^{2,3,5}$ First, it is important to determine a person's sleep habits such as his or her sleep schedule, caffeine and alcohol consumption, and smoking history. Next, it is important to examine whether there are any underlying sleep conditions such as obstructive sleep apnea or restless legs syndrome. Underlying medical or psychiatric issues such as pain or depression must also be explored. Finally, it is important to consider any environmental factors such as noise and light that may be hindering sleep.

\section{What are the risks and benefits of using medication therapy for insomnia?}

Medications may reduce insomnia but may have significant side effects, including thinking difficulties and an increased risk for falls. ${ }^{2,3}$ Some medications such as zolpidem (Ambien) may also lead to sleepwalking., ${ }^{2,3}$ In addition, medications may not treat all the underlying issues of insomnia. This is especially true if obstructive sleep apnea or medical issues are present. ${ }^{2,3}$ Finally, medications may be habit-forming, leading people to become dependent on the medications to help them sleep. ${ }^{2,3}$

The results of this study suggest that some new medications such as daridorexant may be safe and tolerated in older adults. Further study in a larger group of people is needed before daridorexant is approved for general use. We need to better understand if there are any long-term effects. We also need to be sure that the medication does not affect the liver or other organs. We need to know if it interacts with any other medications. A related medication called suvorexant has already been shown to improve sleep and is available by prescription. ${ }^{3}$ Because it also is potentially habit-forming, it is considered to be a controlled substance by the US Food and Drug Administration.

\section{What are some ways I can improve my sleep without medications?}

Cognitive behavioral therapy (CBT) has been helpful in treating insomnia. CBT is a form of talk therapy. It trains people to identify thoughts and behaviors that may in terfere with sleep and modify them. It has been shown to improve sleep in older adults. ${ }^{2,3}$ A specialist trained in CBT can provide exercises to help manage insomnia and stress. There are also web-based programs, books, and mobile device applications that may be more accessible. ${ }^{3}$

Other changes can help., ${ }^{2,3}$ You should maintain a consistent sleep schedule. It is helpful to develop a bedtime routine to prepare yourself for sleep. It is best to eliminate naps during the day and to avoid using caffeine in the late afternoon or evening. Alcohol use before bedtime should be avoided. Additional suggestions include keeping the bedroom for sleep only (for example, do not work in bed). Using electronic devices or watching television before bedtime should be avoided. If you are unable to fall asleep or stay asleep within 20 minutes, it is important go into another room and perform a relaxing activity.

Because treatment options for insomnia may vary and require a comprehensive evaluation, you should talk to your doctor to determine a personalized treatment approach.

\section{For more information}

\author{
Brain \& Life \\ brainandlife.org/ \\ Sleep Education website \\ sleepeducation.org/ \\ National Sleep Foundation \\ sleepfoundation.org/
}

\section{References}

1. Zammit G, Dauvilliers Y, Pain S, Sebök Kinter D, Mansour Y, Kunz D. Daridorexant, a new dual orexin receptor antagonist, in elderly subjects with insomnia disorder. Neurology 2020;94:e2222-e2232.

2. Brewster GS, Riegel $B$, Gehrman PR. Insomnia in the older adult. Sleep Med Clin 2018;13:13-19.

3. Abad VC, Guillenminault C. Insomnia in elderly patients: recommendations for pharmacological management. Drugs Aging 2018;35:791-817.

4. Hirshkowitz M, Whiton $\mathrm{K}$, Albert SM, et al. National Sleep Foundation's sleep time duration recommendations: methodology and results summary. Sleep Health 2015;1:40-43.

5. Ohayon MM. Epidemiology of insomnia: what we know and what we still need to learn. Sleep Med Rev 2002;6:97-111. 


\section{Neurology}

\section{Daridorexant: A new medication for insomnia in older adults? \\ Jennifer Rose V. Molano \\ Neurology 2020;94;e2287-e2289 \\ DOI 10.1212/WNL.0000000000009489}

This information is current as of May 25, 2020

Updated Information \& Services

References

Permissions \& Licensing

Reprints including high resolution figures, can be found at: http://n.neurology.org/content/94/21/e2287.full

This article cites 5 articles, 1 of which you can access for free at: http://n.neurology.org/content/94/21/e2287.full\#ref-list-1

Information about reproducing this article in parts (figures,tables) or in its entirety can be found online at:

http://www.neurology.org/about/about_the_journal\#permissions

Information about ordering reprints can be found online:

http://n.neurology.org/subscribers/advertise

Neurology ${ }^{\circledR}$ is the official journal of the American Academy of Neurology. Published continuously since 1951, it is now a weekly with 48 issues per year. Copyright @ 2020 American Academy of Neurology. All rights reserved. Print ISSN: 0028-3878. Online ISSN: 1526-632X.

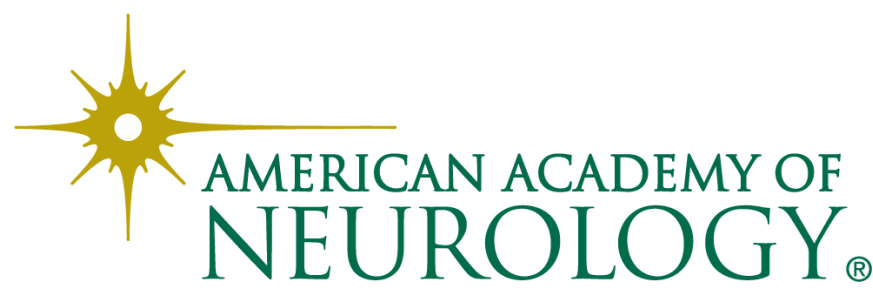

